\title{
Warning: The ECG May Be Normal in Severe Hyperkalemia
}

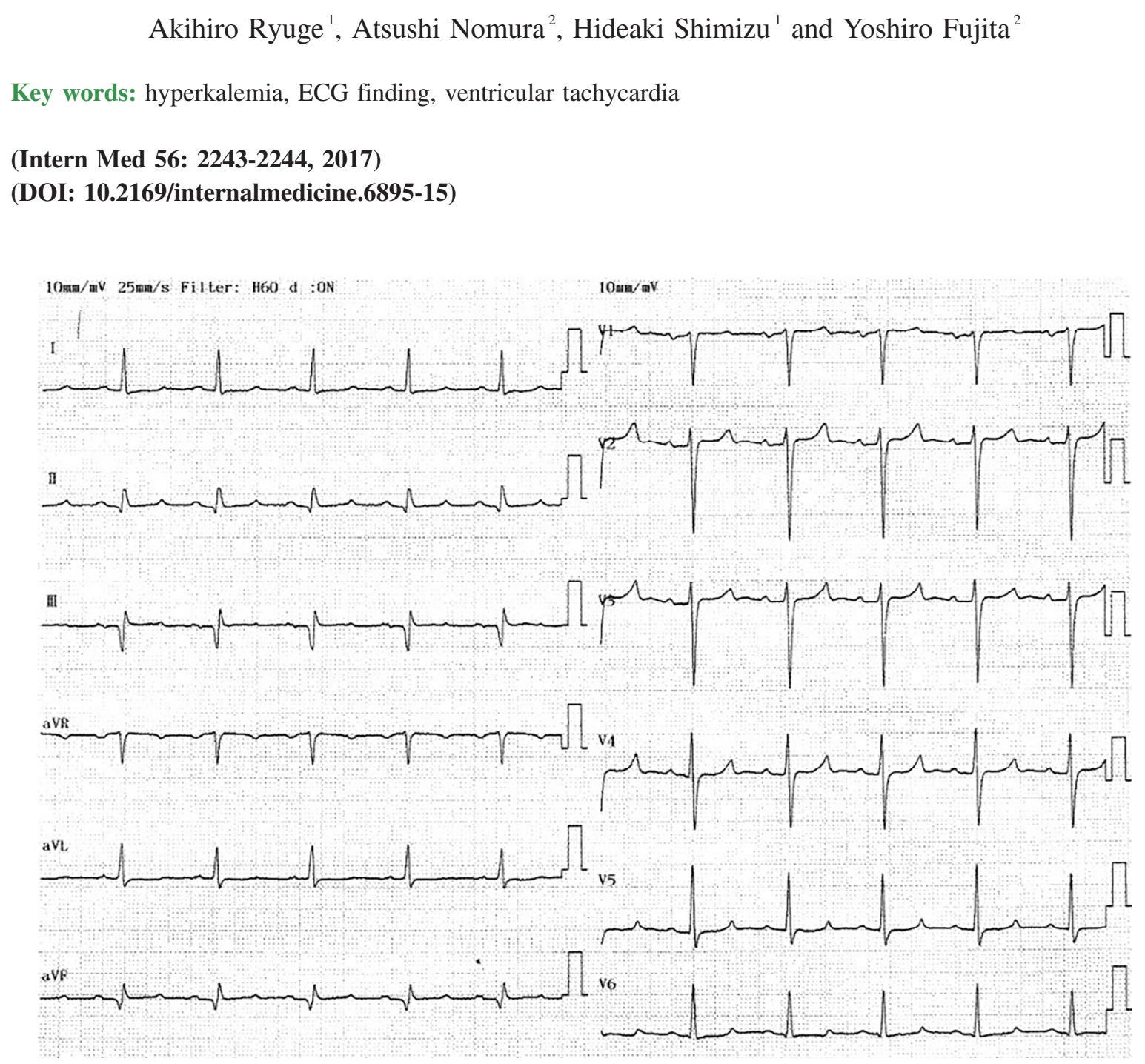

Picture 1.

A 77-year-old man with chronic kidney disease was admitted to our hospital due to acute kidney injury and hyperkalemia (serum potassium, $8.5 \mathrm{mEq} / \mathrm{L}$ ) with a normal ECG finding (Picture 1). Hyperkalemia was caused by tumor lysis syndrome from gastric cancer and medications, including enalapril, carvedilol, and spironolactone. His hyperkalemia improved following the discontinuation of these medications and administration of sodium polystyrene sulfonate. The patient selected best supportive care, rather than chemotherapy, for his cancer and was discharged six days after admission. However, he returned five days later due to the deterioration of his general condition and hyperkalemia (serum potassium, $8.6 \mathrm{mEq} / \mathrm{L}$ ). At this admission, the ECG showed sine-wave ventricular tachycardia (VT) (Picture 2). This case report suggests that ECG changes in hyperkalemia may be established not only by the level of hyperkalemia, but also by the development of hyperkalemia. Therefore, hyperkalemia should not be diagnosed based on ECG findings.

The authors state that they have no Conflict of Interest (COI). 


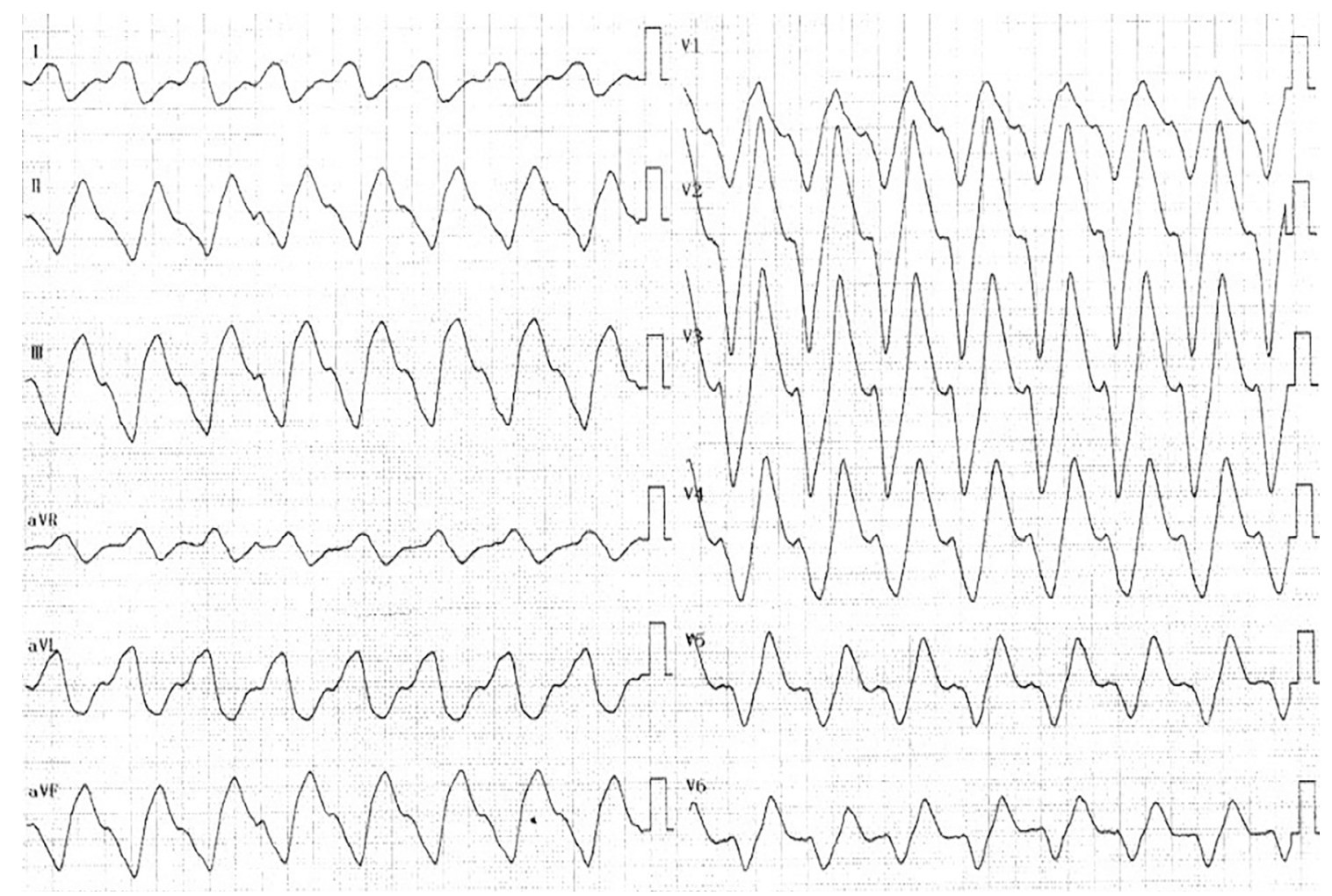

Picture 2.

\section{Acknowledgement}

We express our sincere thanks to Dr. Gerald H. Stein for giving us constructive comments and warm encouragement.
The Internal Medicine is an Open Access article distributed under the Creative Commons Attribution-NonCommercial-NoDerivatives 4.0 International License. To view the details of this license, please visit (https://creativecommons.org/licenses/ by-nc-nd/4.0/).

(C) 2017 The Japanese Society of Internal Medicine Intern Med 56: 2243-2244, 2017 